\title{
Kissing-Watchman technique applied in single-lobulated left atrial appendage anatomy with giant ostia
}

\author{
Linying $\mathrm{Xia}^{1,2 *}$, Yi Liu ${ }^{1 *}$, Ling $\mathrm{Tao}^{1}$ \\ ${ }^{1}$ Department of Cardiology, Xijing Hospital, Fourth Military Medical University, Xi'an 710032, Shaanxi, China \\ ${ }^{2}$ Department of Cardiology, The Second Affiliated Hospital of Xi' an Jiaotong University, Xi' an 710004, \\ Shaanxi, China
}

The only commercially available device in the documented institution is the Watchman (Boston Scientific, Marlborough, MA, USA) occluder which is the predominant occluder in China. The single device approach may not always fit the great anatomic variability of the left atrial appendage (LAA). Adequate closure may require more than a single device. The commercially available Watchman device only fits LAAs with a maximum diameter of $30 \mathrm{~mm}$ [1-3]. In patients with bilobulated LAA, the one-stop implantation of double Watchman is feasible as reported by a previous study [4]. However, it is unknown whether this approach is feasible in the setting of a single-lobulated LAA with a giant ostium $(>30 \mathrm{~mm})$. Reported herein, is a consecutive case series of patients in whom kissing-Watchman was performed to achieve adequate closure of the single-lobulated LAA with large-ostium.

Three out of 100 consecutive patients underwent kissing-Watchman occlusion under the guidance of transesophageal echocardiography (TEE). This experimental procedure was preceded with the patient's acceptance. Before intervention, TEE was performed to exclude thrombi in the LAA. The procedures were performed via femoral access under general anesthesia, and were controlled by the angiography and TEE. At the beginning of the intervention, 5000 units of heparin were given. After transseptal passage, TEE measurement of the LAA diameter at the intended implantation site was performed at four different angles. According to the three cases, the maximal LAA ostia diameters were 35,36 , and $33 \mathrm{~mm}$, respectively.
The first occluder was deemed to cover half of the estimated LAA orifice area $(\mathrm{d} 1=\mathrm{LAAmax} / \sqrt{ } 2)$. Additionally, the size of the first Watchman device should be greater than or equal to the size of the second device. The second occluder was chosen based on residual space. The first Watchman device was pre-released and held still at the intended implantation site (Fig. 1A). After another transseptal puncture via the same femoral site, the pigtail catheter with the second access sheath was delivered carefully to the uncovered space parallel to the first access sheath and contrast injection was made (Fig. 1B). Excessive adjustment was avoided in order to minimize the sheath-sheath interplay. The second Watchman device was chosen according to the residual stump and was carefully placed next to the first device in a kissing fashion. Two Watchman devices were deployed adjacently. Therefore, complete LAA closure was achieved. Fluoroscopy and TEE were used to confirm the position, size and seal of the kissing device. The tugtest was performed on the two devices by pulling the parallel deliver system simultaneously to avoid dislocation (Fig. 1C). The two devices were released respectively after the Position, Anchor, Size and Seal (PASS) criteria had been met (Fig. 1D). The final diameters of each kissing-Watchman deployed were measured by TEE and the maximum device compression ratio of the kissing-Watchman LAA closure should be larger than a single device LAA closure technique (the upper limit was not limited to $30 \%$ ). This higher compression ratio contributed to a more radical deformation of the

Address for correspondence: Ling Tao, MD, PhD, Professor and Chief, Department of Cardiology, Xijing Hospital, Fourth Military Medical University, 15 Changle West Road, Xi'an 710032 China, tel: 86-29-8477-5183, fax: 86-29-8321-1024, e-mail: lingtao@fmmu.edu.cn

Received: 3.04.2019 Accepted: 4.07.2019

*The first two authors contributed equally to this work. 


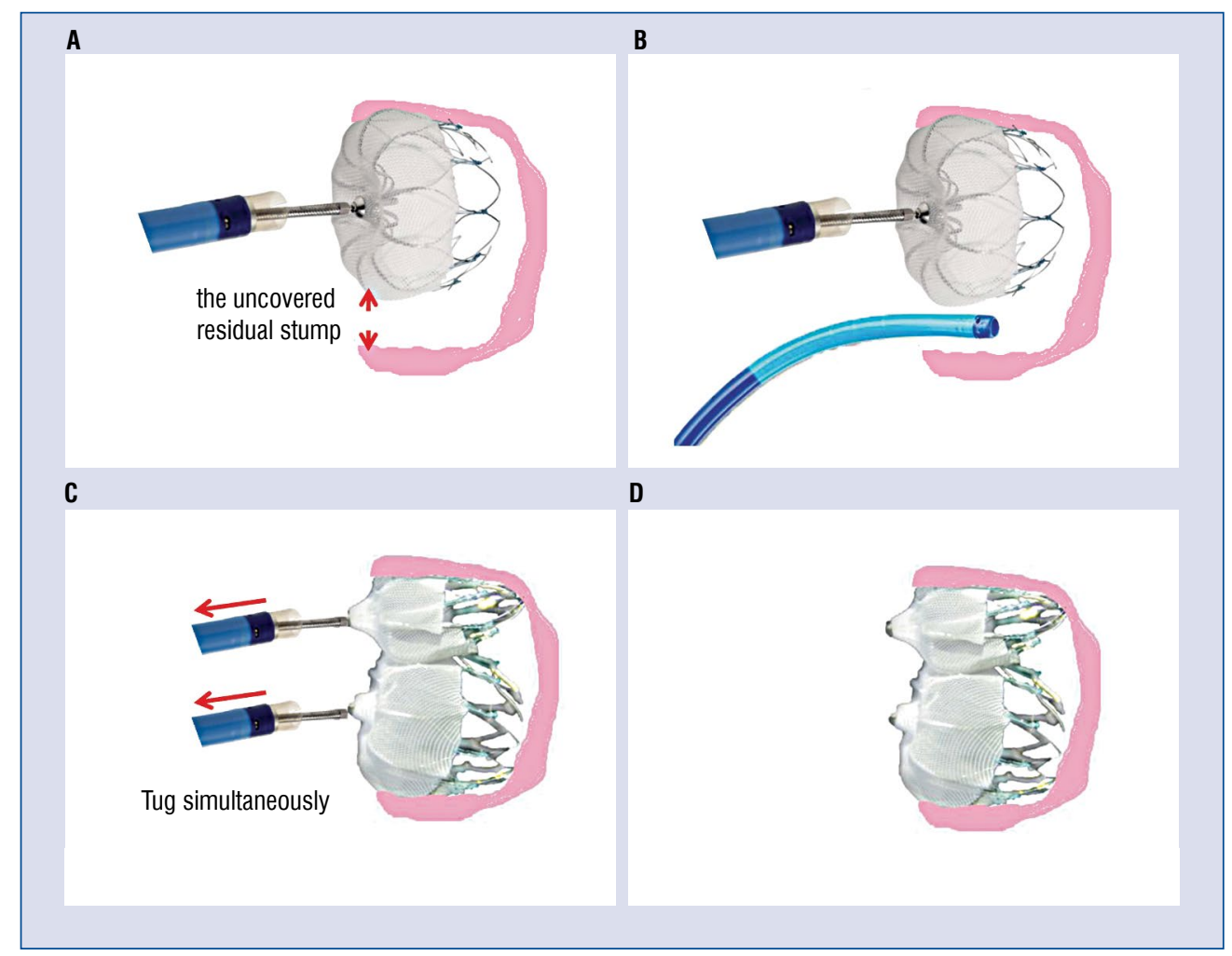

Figure 1. Diagram of kissing-Watchman technique; $\mathbf{A}$. The first device is pre-released and held still at the intended implantation site, leaving a residual stump uncovered; $\mathbf{B}$. The second access sheath is delivered to the uncovered space parallel to the first access sheath; C. The tug test is performed on the kissing-devices simultaneously; D. The two devices are released after the Position, Anchor, Size and Seal (PASS) criteria have been met.

kissing-device on the mutual compression side reducing the possibility of inter-device residual flow. Larger endotheliazation area of the kissing device contributes to prolongation of antithrombotic/antiplatelet therapy (3-month antithrombotic therapy or 12-month antiplatelet therapy). TEE 3-month and 12-month was scheduled after kissingWatchman implantation.

The first patient was a 70-year-old male with a $\mathrm{CHA}_{2} \mathrm{DS}_{2}$-VASc score of 3 . He was referred for LAA closure due to gastrointestinal bleeding on warfarin. TEE showed a single lobe LAA with a maximum LAA ostium of $35 \mathrm{~mm}$ (Suppl. Fig. 1A). In a single intervention, the giant LAA was partially closed by the implantation of a $27 \mathrm{~mm}$ Watchman, and the remaining ostium with a diameter of $12.3 \mathrm{~mm}$ was closed by implantation of another $21 \mathrm{~mm}$ Watchman (Suppl. Fig. 1B). TEE showed that the maximum device compression ratio of the kissing-Watchman LAA closure was $19 \%$ and a complete LAA closure was achieved (Suppl. Fig. 1C, D). The patient was placed on full dose rivaroxaban for 3 months after the intervention and dual antiplatelet therapy daily afterwards.
A TEE performed 3 months later showed good position of both devices, with a newly formed gap of $4.6 \mathrm{~mm}$ (Suppl. Fig. 1E). The patient remained asymptomatic. Twelve months after the intervention, TEE confirmed an adequate occlusion with a remarkably shrinking peri-device flow of $1.8 \mathrm{~mm}$ and no device related thrombus was identified (Suppl. Fig. 1F).

The second patient was a 55-year-old male who underwent closure of a giant single-lobulated LAA due to paroxysmal atrial fibrillation, a $\mathrm{CHA}_{2} \mathrm{DS}_{2}$-VASc score of 4 , and recurrent stroke under warfarin. TEE showed a single lobe LAA with a maximum LAA ostium of $36 \mathrm{~mm}$ without a ridge-like pectinate muscle inside (Suppl. Fig. 2A). Knowing that two devices would be necessary, the giant LAA was partially closed by implantation of a $27 \mathrm{~mm}$ Watchman leaving a $24 \mathrm{~mm}$ opening stump (Suppl. Fig. 2B). Therefore, complete LAA closure was achieved by the subsequent implantation with an additional $27 \mathrm{~mm}$ Watchman (Suppl. Fig. 2C, D). The maximum device compression ratio of the kissing-Watchman system was $33.3 \%$. The patient was discharged on $110 \mathrm{mg}$ dabigatran bid for the 
first 3 months post-operation and dual antiplatelet therapy for the following 8 months. TEE 3 -month and 1-year post-operation follow-up showed adequate LAA sealing, with a persistent residual flow of $1.6 \mathrm{~mm}$ in the LAA and no device related thrombus was identified (Suppl. Fig. 2E, F). One year after the intervention the patient remained free of symptoms on acetylsalicylic acid $100 \mathrm{mg}$ daily indefinitely.

The third patient was a 78-year-old man with a previous stroke, a $\mathrm{CHA}_{2} \mathrm{DS}_{2}$-VASc score of 4 , and chronic kidney disease precluding the use of warfarin. TEE demonstrated a large single-lobulated LAA with a $33 \mathrm{~mm}$ ostium and many pectinate muscles deep inside (Suppl. Fig. 3A). Knowing that two devices would be necessary, $27 \mathrm{~mm}$ Watchman was placed at the entrance of the LAA. It partially occluded LAA with a $14 \mathrm{~mm}$ residual stump uncovered (Suppl. Fig. 3B). A subsequent implantation with an additional $21 \mathrm{~mm}$ Watchman was achieved to completely occlude this large LAA (Suppl. Fig. 3C-E). The maximum compression ratio of the kissing-Watchman system was $28.6 \%$. The patient was prescribed with dual antiplatelet daily for the following 12 months, post-implantation. TEE performed after 3 months showed good position of both devices, with no evidence of residual shunt (Suppl. Fig. 3F). The clinical follow-up was uneventful.

There is a myriad of variations of LAA anatomy in terms of number of lobes and size of orifices [5]. One restriction inherent in single-device LAA occlusion technique concerns the maximum LAA body size suitable for intervention. Though there are larger sizes in other devices nowadays, the largest commercially available Watchman device only fits LAA with a maximum diameter of $30 \mathrm{~mm}$ [1]. Therefore, for an LAA ostium $>31 \mathrm{~mm}$, it is difficult to obtain complete closure with a single Watchman device. The implantation of double Watchman is recommended in bilobulated LAA anatomy [1-3]. It remains controversial whether kissing-device, deployed adjacently in the same lobe is safe. Some scientists argue that there are several potential procedural risks [4]: 1) a severe residual flow between devices can exist; 2) the fixation barbs may injure the permeable polyester polyethylene membrane of the first device while deploying the second device; 3 ) due to the longterm mechanical interaction, device embolization may occur. In a considered opinion, to avoid potential peri-operative complications, it is important to: 1) select the kissing-device of identical or similar size to facilitate closer contact between the two devices minimizing residual shunting between them;
2) a more liberal oversizing technique (higher compression ratio) helps to minimize inter-device flow and also contributes to a greater radial force for stability; 3) deformation of the kissing-device on the mutual compression side contributes to minimizing potential leak between the kissing-devices.

No device dislocation, severe residual flow ( $>5 \mathrm{~mm}$ ) or device related thrombosis was observed, aside from the minor $(<3 \mathrm{~mm})$ peri-device leakage identified at both 3 -month and 12-month TEE follow-up. It was reported that, in patients who had undergone single Watchman LAA closure, an intraprocedural gap could persist, and could close or close and reopen during the follow-up period [6]. Therefore, in patients who underwent kissing-Watchman implantation, a mild peri-device leak during TEE follow-up may not be related to interaction between the devices. Additionally, a peri-device gap with mild blood leak may not affect the stability of the Watchman device inside LAA, as evidenced by the fact that no device dislodgement occurred in patients of the present study.

Due to individual anatomic variations, a single Watchman device may not always adequately seal the LAA. In these cases, implantation of kissingWatchman in single-lobulated LAA with giant ostia may afford a good anatomical result. Larger cohorts are needed to corroborate the safety and efficacy of this technical innovation.

\section{Conflict of interest: None declared}

\section{References}

1. Sick PB, Schuler G, Hauptmann KE, et al. Initial worldwide experience with the WATCHMAN left atrial appendage system for stroke prevention in atrial fibrillation. J Am Coll Cardiol. 2007; 49(13): 1490-1495, doi: 10.1016/j.jacc.2007.02.035, indexed in Pubmed: 17397680.

2. Ernst G, Stöllberger C, Abzieher F, et al. Morphology of the left atrial appendage. Anat Rec. 1995; 242(4): 553-561, doi: 10.1002/ ar.1092420411, indexed in Pubmed: 7486025.

3. Liu Z, Yu J, Fang PH, et al. Double device left atrial appendage closure with the WATCHMAN ${ }^{\mathrm{TM}}$. Int J Cardiol. 2015; 187: 281-282, doi: 10.1016/j.ijcard.2015.03.289, indexed in Pubmed: 25838231.

4. Zhang Y, Zhang J, Chen Y, et al. Double WATCHMAN ${ }^{\mathrm{TM}}$ device left atrial appendage closure in an atrial fibrillation patient with double lobes left atrial appendage (In Chinese). Chin J Cardiac Arrhyth. 2017; 21: 261-262.

5. Jiang L, Duenninger E, Muenzel M, et al. Percutaneous left atrial appendage closure with complex anatomy by using the staged 'kissing-Watchman' technology with double devices. Int J Cardiol. 2018; 265: 58-61, doi: 10.1016/j.ijcard.2018.05.007, indexed in Pubmed: 29754931.

6. Bai R, Horton RP, DI Biase L, et al. Intraprocedural and long-term incomplete occlusion of the left atrial appendage following placement of the WATCHMAN device: a single center experience. J Cardiovasc Electrophysiol. 2012; 23(5): 455-461, doi: 10.1111/j.15408167.2011.02216.x, indexed in Pubmed: 22082312. 